\title{
Ductographic Findings of Breast Cancer
}

\author{
Nariya Cho, MD' \\ Woo Kyung Moon, MD' \\ Sun Yang Chung, MD' \\ Joo Hee Cha, MD' \\ Kyung Soo Cho, MD ${ }^{1}$ \\ Eun-Kyung Kim, MD² \\ Ki Keun Oh, MD²
}

\begin{abstract}
Ductography has become the gold standard for the evaluation of patients exhibiting pathologic nipple discharges. In nine patients (age range, 29-67 years; median age, 51 years) with invasive $(n=5)$ or intraductal $(n=4)$ cancer, ductographic findings were recorded, then correlated with mammographic and sonographic findings. Common ductographic findings included complete ductal obstruction, multiple irregular filling defects in the nondilated peripheral ducts, ductal wall irregularities, periductal contrast extravasation, and ductal displacement. Faint microcalcifications or ill-defined masses, which were not opacified by contrast material, were often discovered adjacent to ductal abnormalities. Mammographically and sonographically occult diffusely spreading intraductal cancers often manifested as pathologic nipple discharge. In such cases, meticulous ductographic examinations and interpretations were crucial in order not to miss breast cancers.
\end{abstract}

\section{Index terms:}

Breast, abnormalities

Breast, ducts

Breast neoplasms, diagnosis

Galactography

\section{Korean J Radiol $2005 ; 6: 31-36$}

Received September 14, 2004; accepted after revision January 4, 2005.

'Department of Radiology, Seoul National University College of Medicine, Institute of Radiation Medicine, SNUMRC, and Clinical Research Institute, Seoul National University Hospital; ${ }^{2}$ Department of Radiology, Yonsei University College of Medicine

Address reprint requests to:

Woo Kyung Moon, MD, Department of Diagnostic Radiology, Seoul National University Hospital, 28 Yongon-dong, Chongno-gu, Seoul 110-744, Korea.

Tel. (822) 2072-1862

Fax. (822) $747-7418$

e-mail: nariya@radiol.snu.ac.kr
$\mathrm{P}$ athologic nipple discharge is defined as unilateral, single-pore, spontaneous discharge. About $8-15 \%$ of pathologic nipple discharges are caused by underlying breast cancer, although intraductal papilloma constitutes the most common cause of this phenomenon $(1,2)$. Ductography remains the gold standard for the evaluation of patients exhibiting pathologic nipple discharges. This technique allows for the diagnosis of the underlying conditions, the definition of disease extent, and guidance for surgical excision (1-3).

However, some radiologists maintain that ductography is prohibitively time-consuming, and that it can be replaced, at least in part, by sonography (4-6). Intraductal papillary lesions are sometimes visualized as isoechoic or hyperechoic masses in dilated ducts on sonography, and can often be biopsied under sonographic-guidance. However, many cases of sonography, even when combined with spot magnification mammography, fail to reveal the underlying causes of pathologic nipple discharge, as the lesions are frequently too small, contain no calcifications, or prove to be completely intraductal (2).

Ductography should be performed precisely, and interpreted meticulously, so as not to miss important signs of breast cancer, and to avoid delayed diagnosis. Previous articles have extensively reviewed ductographic techniques, and reported on the nonspecific findings of benign and malignant diseases which can be responsible for nipple discharge $(1,3,7)$. In this pictorial essay, we illustrate the various ductographic appearances of breast cancer, when manifesting as a pathologic nipple discharge, from obviously suspicious findings to subtle findings which are often overlooked. 
Cho et al.

\section{PATIENT SELECTION}

Between November 1999 and March 2002, 45 consecutive women exhibiting pathologic nipple discharge underwent ductographies. Twenty-four women underwent excisional or percutaneous needle biopsies due to abnormal ductographic findings. Among these 24, nine women (age range, $29-67$ years; median age, 51 years) were diagnosed with various breast cancers, and subsequently included in this study. One of these women was diagnosed with infiltrating ductal carcinoma, usual type, one with invasive papillary carcinoma, three with ductal carcinoma in situ with focal invasions, and four were diagnosed with pure ductal carcinoma in situ. All of these patients presented with bloody $(8 / 9,89 \%)$ or clear $(1 / 9,11 \%)$ nipple discharge, and the absence of palpable masses.

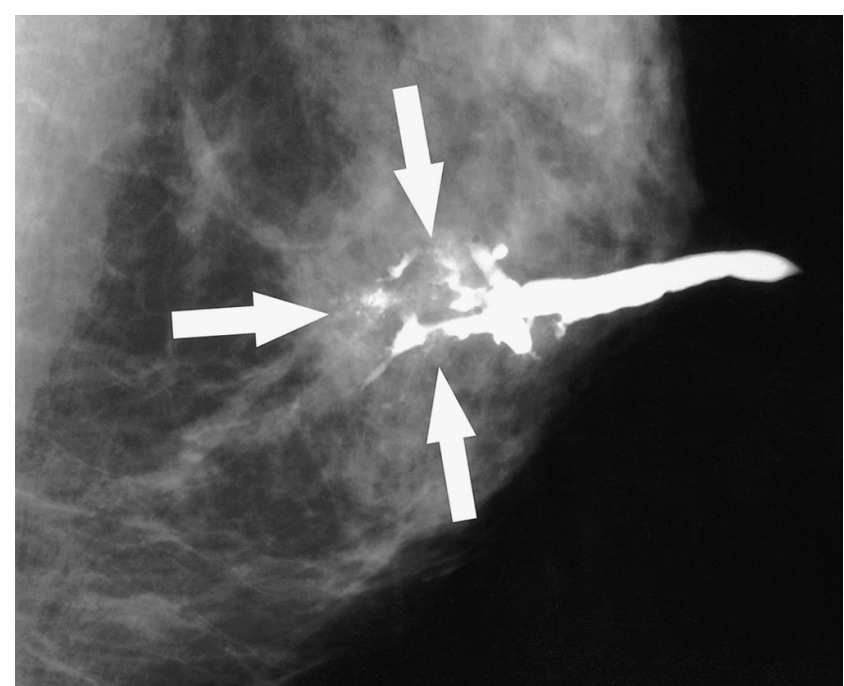

Fig. 1. A 52-year-old woman with a $2.2 \mathrm{~cm}$ ductal carcinoma in situ with microinvasion who presented with bloody nipple discharge. The mediolateral oblique ductogram reveals complete obstruction with a distal, irregular, moth-eaten appearance (arrows), and associated microcalcifications.
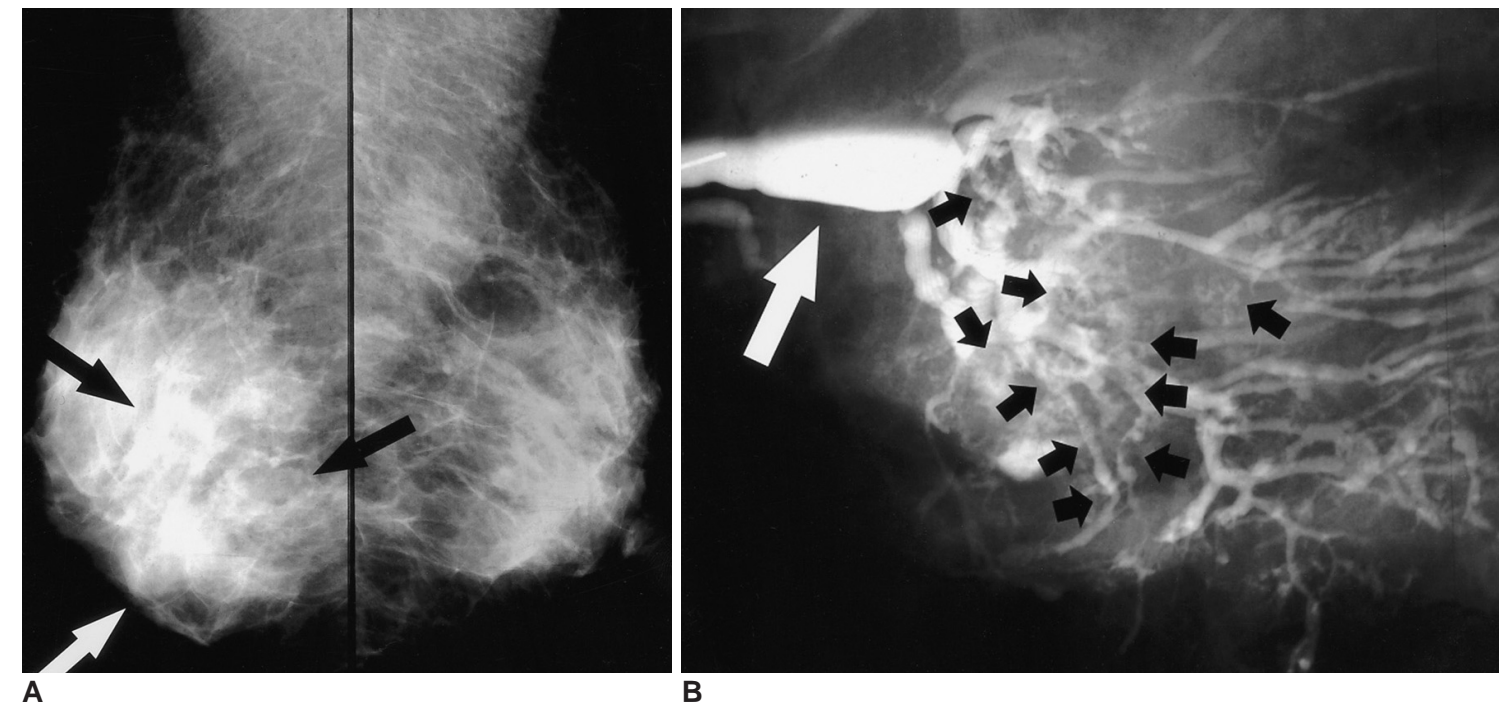

Fig. 2. A 48-year-old woman with a $3 \mathrm{~cm}$ invasive papillary carcinoma who presented with clear nipple discharge. A. Mediolateral oblique mammograms of both breasts reveal illdefined diffuse asymmetric increased density in the lower region of the right breast (arrows).

B. Mediolateral oblique ductogram reveals innumerable oval and irregular-shaped filling defects (black short arrows) below the segmental ducts in the right lower region. Dilatation of the lactiferous sinus can also be observed (white arrow).

C. Sonogram reveals an ill-defined, heterogeneously hypoechoic area (arrows) with no delineated masses in the right 6 o'clock to 10 o'clock area. 


\section{DUCTOGRAPHIC TECHNIQUES}

Before cannulation, mediolateral and craniocaudal views were obtained, in order to assess suspicious microcalcifications or nodules. A light bulb was focused on the nipple for 15 minutes, in order to relax the periareolar sphincter muscle. With the patient in a sitting position, the nipple and periareolar area were sterilized with an alcohol swab, after the application of a povidone-iodine swab. Gentle periareolar pressure was then applied, in order to locate the trigger point. Cannulation, targeting the trigger point, was performed with a 24-gauge intravenous catheter (Angiocath; Becton Dickinson Infusion Therapy Systems, Sandy, Ut.), attached to a $1 \mathrm{~mL}$ tuberculin syringe filled with telebrex meglumine (Telebrex 30; Guerbet, Paris, France). As the tip of the catheter descended into the nipple duct, which indicates proper cannulation, the contrast agent was slowly injected until the patient reported feeling pain, or the operator perceived resistance. Immediately upon the discontinuation of injection, the catheter was removed, and a craniocaudal subareolar magnification view was obtained in order to verify the intraductal filling of the contrast agent. The contrast agent was subsequently expelled from the duct by the applica- tion of gentle periareolar pressure. For more detailed images, recannulation, reinjection and various lesion approach mammography were performed. This examination normally requires about $20-30$ minutes.

\section{DUCTOGRAPHIC FINDINGS}

\section{Complete Ductal Obstruction}

Complete ductal obstruction is not pathognomonic of breast cancer, and can be observed in both benign and malignant tumors. This finding was noted in $5-47 \%$ of benign diseases, and in $67-83 \%$ of cancers, by ductography $(7,8)$. In approximately $70 \%$ of obstructing papillomas, contrast material was observed to partially outline the leading edge of a lesion, resulting in a meniscus-like appearance $(7,8)$. By way of contrast, the shape of the cut-off site in the carcinoma on ductography often assumes an irregular, moth-eaten appearance (Fig. 1). According to these reports, this moth-eaten appearance was observed in $56-$ $76 \%$ of cancers $(8,9)$. In addition, an irregular-shaped mass is often observed at the point of malignant obstruction.

\section{Multiple Filling Defects}

Multiple irregular filling defects in nondilated peripheral ducts are highly suggestive of malignancy (Fig. 2). Seventy

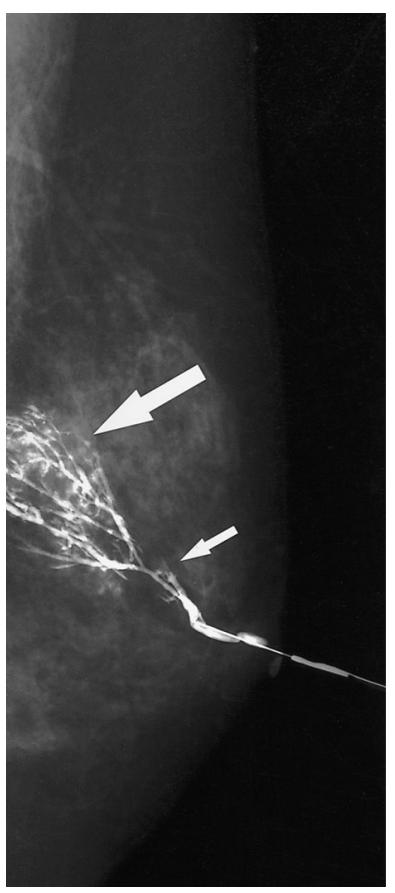

A

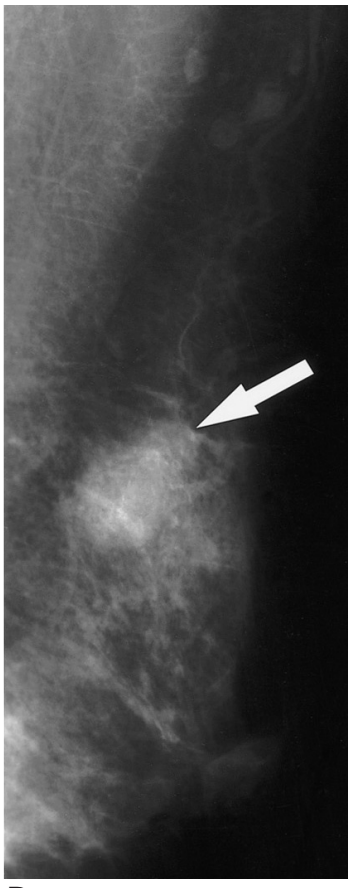

$B$

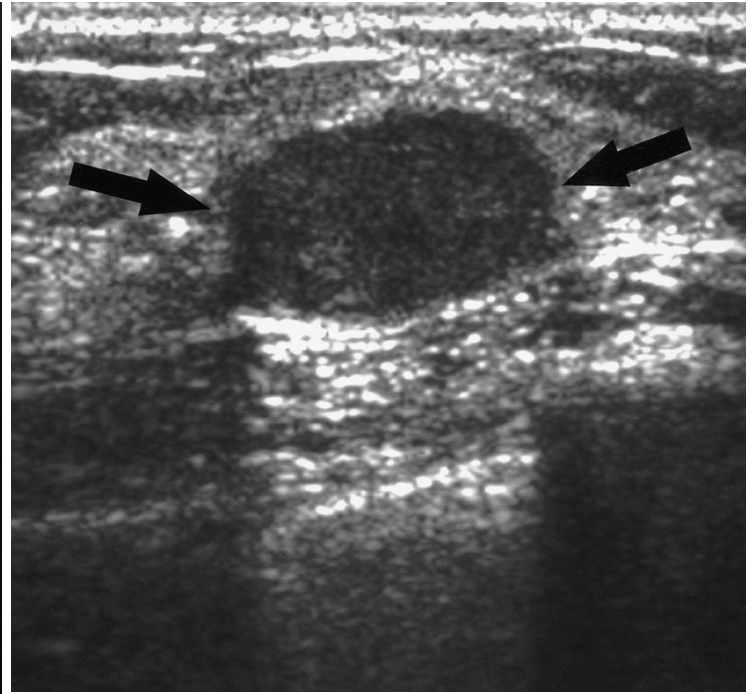

C

Fig. 3. A 44-year-old woman with bloody nipple discharge.

A. Initial mediolateral ductogram reveals focal ductal wall irregularities below the subsegmental ducts in the peripheral portion (long arrow) of the left breast. This finding was overlooked, and the only reported abnormal finding was a questionable cut-off lesion in the segmental duct (short arrow).

Sonography was negative (not shown here). Duct excision targeting the cut-off lesion was performed, and fibrocystic changes were reported. Subsequently, the bloody nipple discharge ceased.

B. Eighteen months later, this patient revisited the hospital due to a palpable mass in the left breast. Mediolateral oblique mammogram reveals a $2-\mathrm{cm}$ round, partly obscured mass in the palpable area (arrow).

C. Sonogram reveals a 2-cm circumscribed hypoechoic mass (arrows) in the palpable area. US-guided core biopsy revealed an invasive papillary carcinoma. 


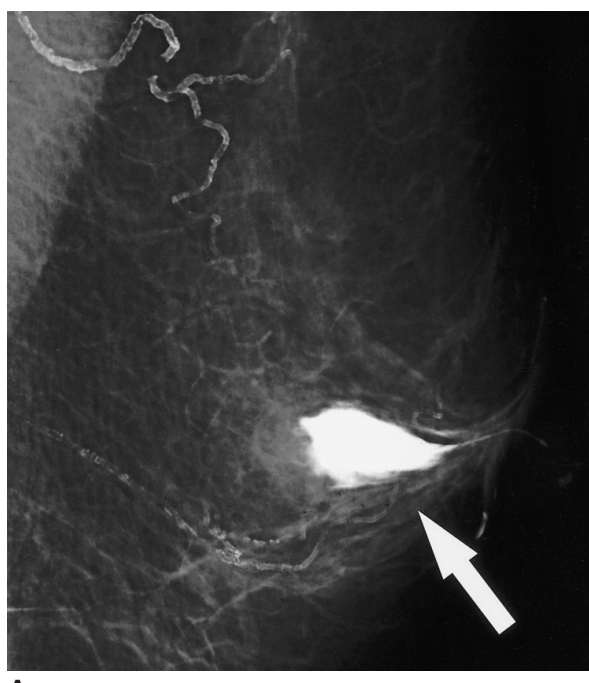

A

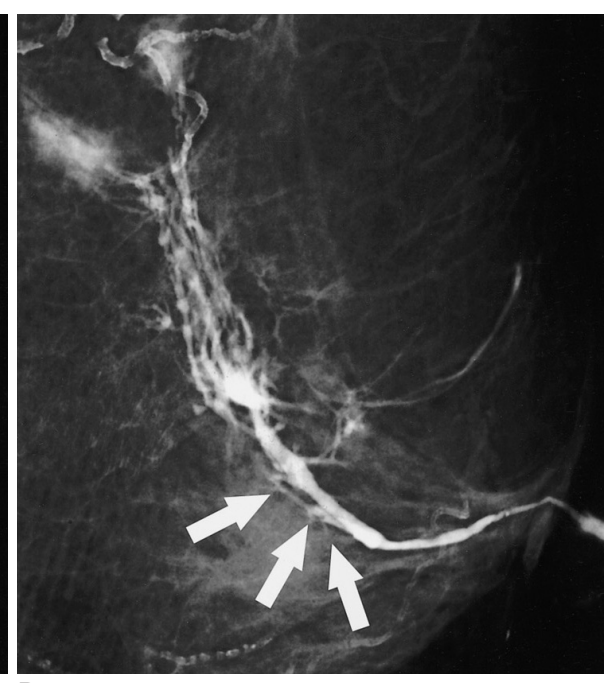

B
Fig. 4. A 67-year-old woman with $0.8 \mathrm{~cm}$ ductal carcinoma in situ, presenting with bloody nipple discharge.

A. The initial mediolateral oblique ductogram reveals contrast extravasation (arrow) in the subareolar area of the left breast.

B. Twelve days later, this repeat mediolateral oblique ductogram reveals ductal obstruction (arrows) and nonopacified subsegmental ducts in the previously extravasated site.

C. Sonogram targeting the subareolar area reveals a $0.5 \mathrm{~cm}$ hypoechoic nodule (arrows) in the dilated duct. In view of the suspicious ductogram findings, a duct excision was performed. The lesion proved to be comedo ductal carcinoma in situ, with pagetoid involvement of the preexisting intraductal papilloma.

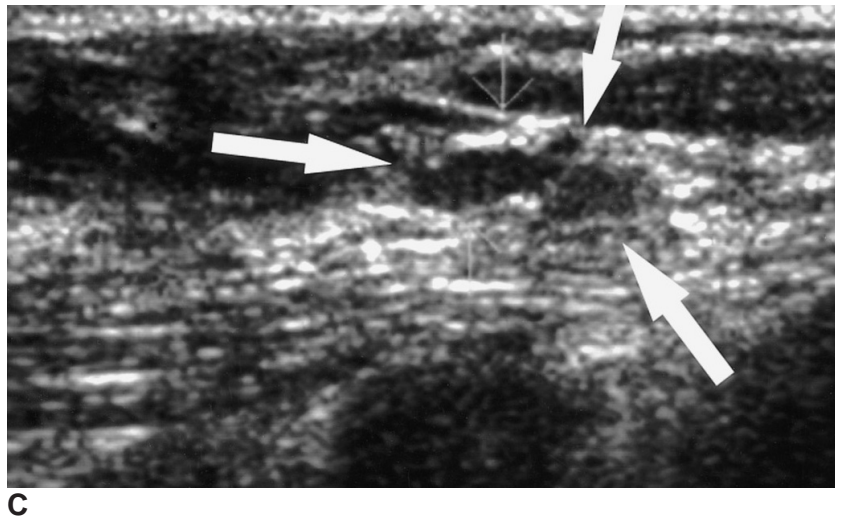

percent to $89 \%$ of previously-reported cases of multiple irregular filling defects proved to be cancerous $(8,9)$ Although peripherally located papillomas also exhibit multiple intraductal filling defects, they tend to have a smooth or lobular surface, and are often coupled with ductal dilatation $(8,9)$. In breast cancers, the main pathology of multiple filling defects visualized by ductography is ductal carcinoma in situ (DCIS), or the extensive intraductal component of invasive carcinoma. Ductal stiffening, distortion, and disruption are often observed when an intraductal cancer invades the stroma. In ductograms, cysts which communicate with the discharging ductal system may be filled. Cysts with filling defects, however, should always be excised, as intraductal or invasive cancers are sometimes associated with intracystic abnormalities (3).

Diffusely involved intraductal cancers with microscopic invasive components might be associated with the triggering of bloody nipple discharge. In these cases, palpable abnormalities are frequently absent, and mammography produces negative findings, or a finding of nonspecific asymmetric increased density (Fig. 2A). When subjected to sonography, these lesions often exhibit a diffusely ill- defined hypoechoic area, without definitively delineated mass formations (Fig. 2C). Even an experienced radiologist is likely to overlook such diffusely spreading breast cancers, owing to their low contrast with the surrounding tissue upon sonography. Ductography, however, can be used to determine the nature and extent of this lesion type, and to facilitate appropriate surgical management.

\section{Ductal Wall Irregularities}

Ductal wall irregularities, as visualized by ductography, represent a nonspecific finding, and may be detected in both papillomas and DCIS. In a previous study, $2.7 \%$ of papillomas and $5.7 \%$ of DCIS were found, by ductography, to be associated with ductal wall irregularities (8). These entities are normally diffuse, and tend to be associated with other suspicious findings. Sometimes, only a focal ductal wall irregularity is discovered at the periphery of the breast in patients with nipple discharges. This can result in false negative ductographic results, or can delay a diagnosis of malignancy (Fig. 3) (3).

If ductal discharge is encountered and treated by central duct excision without intervening ductography, under- or overexcision becomes a real risk; standard central duct excision results in the resection of tissue approximately 4-5 $\mathrm{cm}$ directly posterior to the nipple. As the distal ducts have been transected, discharge ceases, yet the phenomenon underlying the discharge remains (3). In such cases, the residual pathology can become a palpable mass (Fig. 3). Therefore, aggressive approaches, such as ductographyguided wire localization, sometimes become necessary in cases of obvious pathologic nipple discharge (10).

\section{Periductal Contrast Extravasation}

Extravasation is normally the result of forceful contrast 
material administration or wall perforation, as the result of overzealous cannula insertion. On occasion, the destruction of ductal integrity by a carcinoma can also lead to extravasation $(3,7)$ (Fig. 4). When extravasation occurs as the result of an underlying ductal lesion, the patient does not experience pain during cannulation, or burning when contrast material is injected (7). Ductography should, in such cases, be rescheduled for $7-14$ days after the initial extravasation, and the lesion should be considered suspicious in cases in which extravasation is repeatedly detected at the same foci, in the absence of traumatic cannulation.

\section{Displacement of Otherwise Normal Ducts}

Ductal displacement should be considered evidence of a space-occupying lesion, and is not pathognomonic of breast cancer $(7,8)$. However, asymmetric increased density or architectural distortion on mammograms, or ill-defined

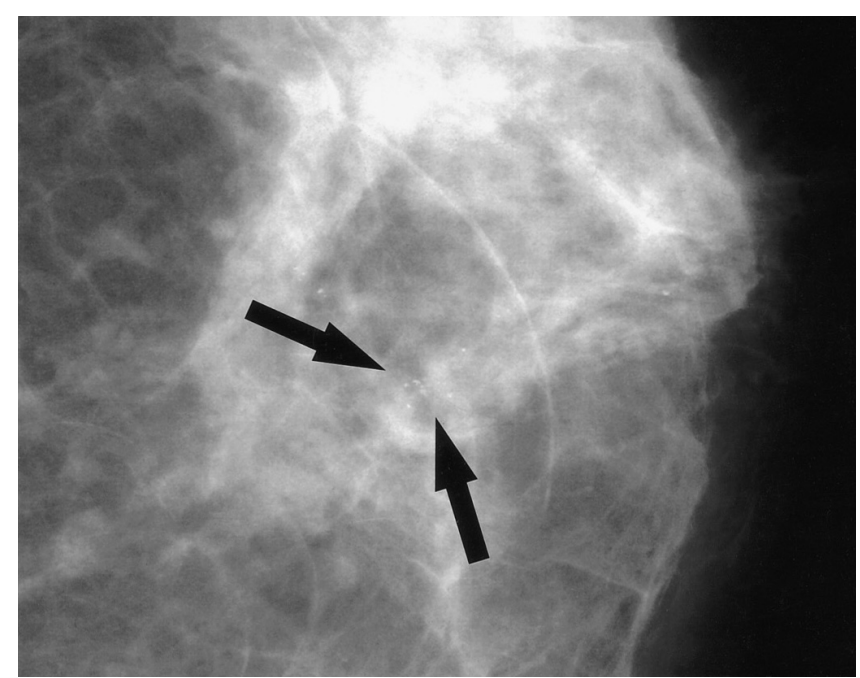

A

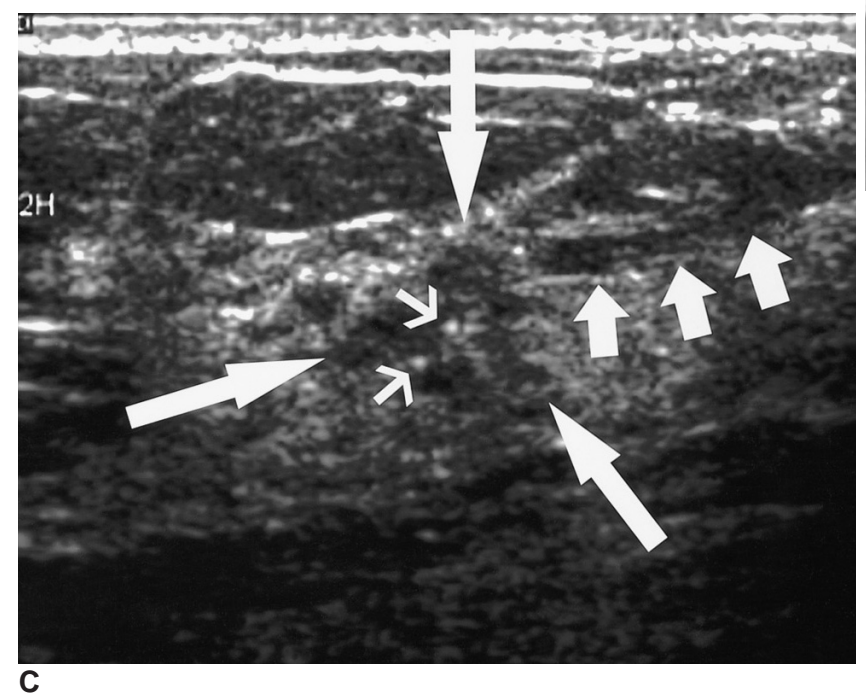

distorted ducts with posterior shadowing on sonography in the corresponding area of ductal displacement on ductograms, both are fairly reliable indicators for the presence of DCIS. Thus, the evaluation of both the morphological features of the opacified ducts, as well as their orientation within the breast, are crucial for adequate diagnosis and treatment.

\section{Microcalcifications or Mass Adjacent to Abnormal Ducts}

Additional evidence of cancer may be detected in tissues adjacent to abnormal ducts, via ductography (3). Faint microcalcifications or ill-defined masses, which are not opacified by the contrast material, are often found adjacent to ductal abnormalities. These microcalcifications or masses are sometimes faint or subtle, and can normally only be detected by spot magnification mammography. These findings could easily be overlooked until ductogra-

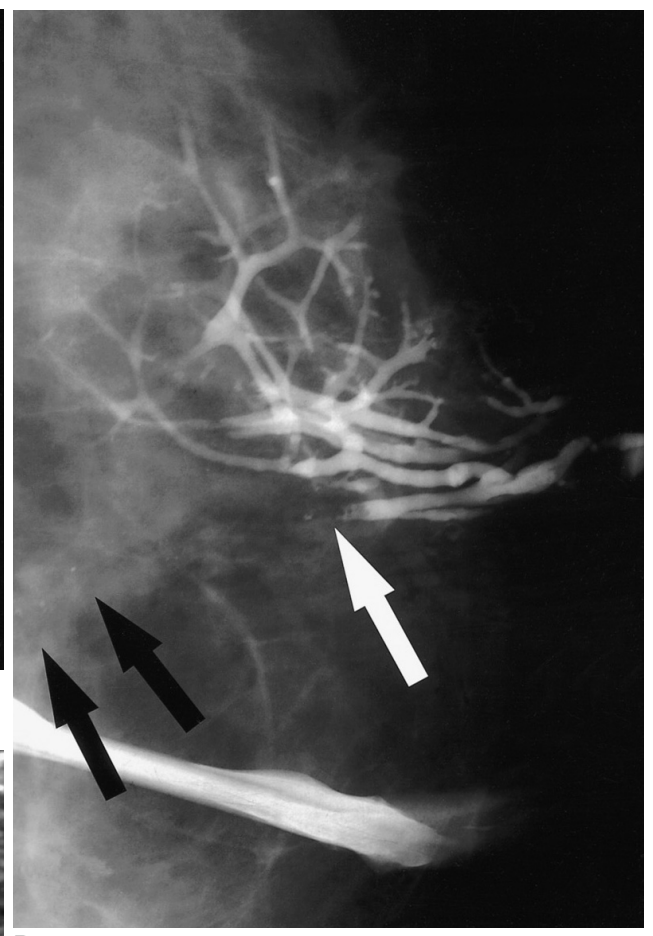

B

Fig. 5. A 49-year-old woman with $5 \mathrm{~cm}$ papillary ductal carcinoma in situ with a $0.5 \mathrm{~cm}$ invasive component, presenting with bloody nipple discharge.

A. Craniocaudal mammogram reveals faint clustered microcalcifications (arrows) without definite mass in the central portion of the patient's left breast.

B. Craniocaudal ductogram reveals complete ductal obstruction (white arrow) with microcalcifications (black arrows).

C. Sonogram targeting the obstructing lesion reveals a $0.5 \mathrm{~cm}$ sized, irregularly shaped, microlobulated hypoechoic mass (long arrows) with internal microcalcifications (thin arrows). Mild dilatation of the subareolar duct was observed (short arrows). 


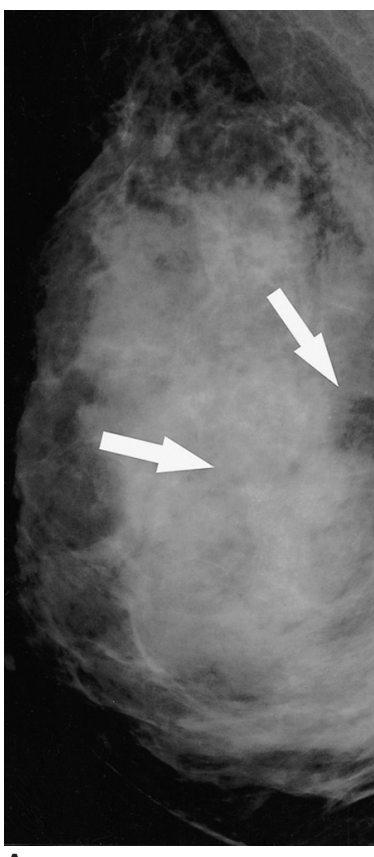

A

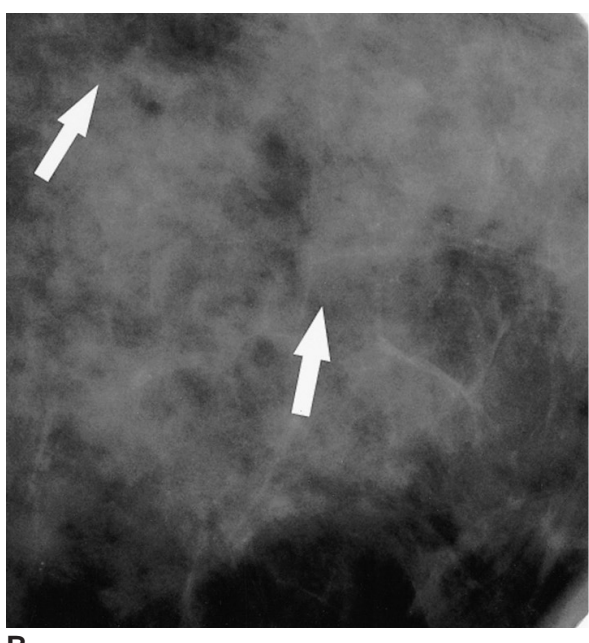

B

Fig. 6. A 41-year-old woman with $8.3 \mathrm{~cm}$ DCIS with a $0.3 \mathrm{~cm}$ invasive component who presented with bloody nipple discharge.

A. Mediolateral mammogram reveals two groups of clustered microcalcifications (arrows) with no palpable mass in the right breast.

B. Spot magnification mammogram demonstrates faint pleomorphic microcalcifications (arrows).

C. Contrast-enhanced, sagittal, three-dimensional, fast spoiled gradient-echo subtraction (postcontrast image minus precontrast image) MR images (TR/TE,19.7 ms/1.6 ms) demonstrate multifocal clumped enhancements, extending from deep in the breast, to the subareolar area. phy is performed, and the radiologist's attention is drawn to this area (Fig. 5).

In patients with nipple discharge, MR imaging can also be used to determine the location and extent of an intraductal lesion $(11,12)$. Ductal carcinoma in situ is often visualized as ductal or segmental clumped enhancements on contrast-enhanced MR images (Fig. 6). The role of MR imaging in the evaluation of patients with nipple discharge should be a focus of future research.

\section{CONCLUSION}

In order to thoroughly and accurately evaluate patients exhibiting pathologic nipple discharges, it is important to perform ductography, and not to miss the subtle but suspicious ductographic findings associated with breast cancer. Diffusely spreading intraductal cancers, with or without focal invasions, are often found to be negative by mammography and sonography. In such cases, ductography constitutes the best imaging method, as it has proven effective in the determination of the nature and extent of such lesions, and can facilitate appropriate surgical management.

\section{References}

1. Tabar L, Dean PB, Pentek Z. Galactography: the diagnostic procedure of choice for nipple discharge. Radiology 1983;149:31-38
2. Sickles EA. Galactography and other imaging investigations of nipple discharge. Lancet 2000;356:1622-1623

3. Slawson SH, Johnson BA. Ductography: how to and what if? RadioGraphics 2001;21:133-150

4. Stavros AT. Nontargeted indications: breast secretions, nipple discharge, and intraductal papillary lesions of the breast. In: Stavros AT, ed. Breast ultrasound, 1st ed. Philadelphia, Pa: Lippincott Williams \& Wilkins, 2004;157-198

5. Moon WK, Myung JS, Lee YJ, Park IA, Noh DY, Im JG. US of ductal carcinoma in situ. RadioGraphics 2002;22:269-280

6. Chung SY, Lee KW, Park KS, Lee Y, Bae SH. Breast tumors associated with nipple discharge. Correlation of findings on galactography and sonography. Clin Imaging 1995;19:165-171

7. Cardenosa G, Doudna C, Eklund GW. Ductography of the breast: technique and findings. AJR Am J Roentgenol 1994;162:1081-1087

8. Hou MF, Huang TJ, Liu GC. The diagnostic value of galactography in patients with nipple discharge. Clin Imaging 2001;25:7581

9. Cho N, Oh KK, Cho HY. Galactographic differentiation between malignant and benign disease in patients with pathologic nipple discharge. J Korean Radiol Soc 2003;48:511516

10. Chow JS, Smith DN, Kaelin CM, Meyer JE. Galactographyguided wire localization of an intraductal papilloma. Clin Radiol 2001;56:72-73

11. Orel SG, Dougherty CS, Reynolds C, Czerniecki BJ, Siegelman ES, Schnall MD. MR imaging in patients with nipple discharge: initial experience. Radiology 2000;216:248-254

12. Nakahara H, Namba K, Watanabe R, Furusawa H, Matsu T, Akiyama F, et al. A comparison of MR imaging, galactography and ultrasonography in patients with nipple discharge. Breast Cancer 2003;10:320-329 